\title{
COVID-19 and Social Isolation Endangering Psychological Health of Older Adults: Implications for Telepsychiatry
}

\author{
Kavita Batra ${ }^{1,2, *}$, Amanda Elizabeth Morgan ${ }^{1}$, Manoj Sharma ${ }^{1}$
}

\author{
${ }^{1}$ Department of Environmental and \\ Occupational Health, School of Public \\ Health, University of Nevada, Las Vegas, \\ Nevada, USA \\ ${ }^{2}$ Department of Biological \\ Sciences, College of Southern Nevada, \\ Las Vegas, Nevada, USA

\section{*Correspondence} \\ drkavita.juneja@gmail.com \\ (Kavita Batra)
}

\begin{abstract}
Coronavirus disease-19 (COVID-19) has created a public health emergency and had claimed 911,877 lives at the time of writing this manuscript (September 12, 2020). While everybody is at the risk of acquiring infection following the exposure to SARS-CoV-2, older adults are significantly more likely to experience severe illness, life-threatening complications, hospitalizations, and deaths. As the pandemic evolved, social distancing or shielding efforts as primary prevention were instituted to protect the physical health status of the vulnerable population, with no or limited consideration to the psychological health of the older population. Social isolation and loneliness are the long-standing public health concerns among older adults, which appear to be exacerbating amidst the COVID-19 pandemic. Given the pre-existing risk of late-life psychiatric disorders among older individuals, it is critical to determine challenges posed by COVID-19 and associated social distancing protocols as it relates to the psychosocial well-being of the aging population. Such information is imperative to design targeted interventions to fulfill the unmet needs of the older population. Therefore, this review endeavors to identify the healthcare and emotional needs of the aging population during the COVID19 pandemic in the context of psychological health and social capital. Additionally, this review also identifies the barriers in the uptake of telepsychiatry and highlights the need for promoting remote counseling services among older people to promote their psychosocial well-being.
\end{abstract}

\section{Keywords}

Social isolation, Loneliness, Older adults, Psychological health, COVID-19, Telepsychiatry

\section{Introduction}

Coronavirus disease-19 (COVID-19) has created a global public health emergency, and as of September 12, 2020 (at the time of writing commentary), it has claimed 911,877 lives [1, 2]. COVID-19 has taken a significant toll across all demographic groups, with the older population being disproportionately affected [2-4]. The increased vulnerability of the older adults toward COVID-19 attributes to several factors, including impaired immune response, anatomic and physiologic changes, the presence of pre-existing conditions, and environmental factors [4-8]. Globally, nearly $66 \%$ of older people aged 70 years or above have at least one pre-existing illness, which increases their susceptibility to acquiring COVID-19. Once infected, approximately $20 \%$ of this population undergo hospitalizations [7]. The risk of infection may further increase for those living in the institutions; for instance, older individuals residing in the nursing homes or long-term care facilities (LTCFs) (institutionalized older adults) are more susceptible to infection compared to community-dwelling [8-10]. LTCFs offer a favorable environment for the transmission of COVID-
19 due to overcrowding, lack of hygiene, and inadequate supervision or testing $[11,12]$. Besides, residents of LTCFs may need assistance with their daily activities, which puts them in proximity to the caregiver, thereby increasing exposure to the virus. According to a recent study of 19 LTCFs in California (United States), 1 in 4 residents of skilled nursing and assisted living facilities were asymptomatic carriers [11, 12]. In the U.S., the number of COVID-19 associated hospitalizations increased with age, with over 500 hospitalizations per 100,000 population among older adults aged above 85 years [3, 4]. Nearly $80 \%$ (8 in 10) of the COVID-19 associated deaths occurred among older adults aged above 65 years in the U.S. $[3,4]$. The case fatality rate of the U.S. older adults aged above 85 years ranges from $10 \%$ to $27 \%$ [3-5]. According to the Joint WHO-China fact-finding mission [6], the overall case fatality rate of COVID-19 in China declined by nearly $96 \%(17.3 \%$ to $0.7 \%)$ from January 2020 to February 2020. In contrast, the case fatality rate among older adults aged above 80 years increased to $21.9 \%[5,6]$. Higher case fatality rates due to COVID-19 were reported among older adults with pre-existing conditions, such as cardiovascular disease, 
hypertension, diabetes, chronic obstructive pulmonary disease, cancer, and chronic kidney disease [3, 4, 6, 7]. Notably, every 1 in 5 individuals has at least one pre-existing condition, of which the older population constitutes a sizable proportion in the U.S. [3, 4, 7].

Besides these chronic ailments, lack of compliance to practice protective behaviors among older adults has recently been discovered in a study of 72,417 participants across 27 countries [13]. These unexpected finding (when one would assume high responsibility attitude from older adults) provides another viewpoint and essential basis to design interventions, which should aim to increase knowledge and awareness among older adults during the pandemic.

Like other demographic groups, the spectrum of illnesses among the older population is not only limited to physical health but also extends to psychosocial health outcomes during the COVID-19 pandemic. As the pandemic evolved, several public health primary prevention measures, including curfews, lockdowns, and physical distancing (sometimes called "shielding"), were instituted globally to minimize the COVID-19 transmission [14, 15]. While shielding is an effective strategy to limit the burden of COVID-19, it comes at the expense of social connectedness [14, 15]. There is consistent evidence that prolonged social isolation and poor social relationships are associated with adverse psychological health outcomes [14-19]. Although these associations hold for all population groups, older adults are significantly more vulnerable, given the background risk of decline in physical, social, and psychological well-being (PWB) with the advancing age [15-22]. Poor or inadequate social support system and perceived isolation among older individuals increase their risk of maladaptive behaviors and mental health disorders, including emotional exhaustion, anxiety, depression, stress, insomnia, low sleep quality, dementia, substance abuse, and suicidal ideation [1522].

Given the pervasive effects of social isolation and a preexisting risk of late-life psychiatric morbidities among older adults, it is essential to determine risk factors, challenges, and barriers in preserving the social connectedness of older adults, as prolonged social isolation may result in exaggeration of adverse psychological health outcomes in this population group during the pandemic and post-pandemic periods [15,22]. Such information is imperative to design early interventions aimed to fulfill the unmet social and emotional needs of the older population, in the context of improving psychosocial health. Despite being in the COVID-19 pandemic for several months, telepsychiatry has not been adequately utilized as primary and secondary prevention of psychiatric morbidities among older adults. Therefore, this commentary aims to identify the impact of COVID-19 and subsequent shielding efforts on social integration of the older adults for determining immediate social and emotional needs in the context of improving psychological health and social capital through telepsychiatry and remote counseling interventions.

\subsection{Shielding, social isolation, loneliness, and psychological health}

COVID-19 containment measures, including the shielding strategies, are significantly more aversive for older adults $[19,20]$. The effect of shielding induced social isolation can be explained by two core constructs of psychological wellbeing (PWB), including purpose in life and personal growth $[23,24]$. Aging involves a decline in these constructs, which may translate into social isolation and loneliness [23, 24]. Social isolation is an objective state of being physically separated from others, whereas loneliness is perceived or subjective isolation [19, 25]. In other words, living alone does not mean social isolation. At the same time, it is possible for someone to feel alone (loneliness) despite being surrounded by people [14]. In the U.S., nearly one-fourth of the older population live alone, but they are not socially isolated [19]. Social isolation and loneliness are among the main drivers of physical and psychosocial health outcomes among older adults $[25,26]$. However, unique contributions to social isolation and loneliness remain equivocal $[20,21]$. Loneliness is a long-standing public health concern among older populations and poses a comparable mortality threat as other well-established risk factors, such as smoking, physical inactivity, and obesity [25]. Loneliness can alter the cell mechanisms to promote inflammation, thereby increasing the susceptibility to infections [19]. Individuals with robust social integrations and supporting web are more likely to have better survival and emotional well-being compared to those being socially disconnected or lonely [27, 28]. Additionally, prolonged duration of loneliness has elevated psychological symptoms, substance abuse, and suicide rates among older adults [27, 28].

\subsection{Stressors and contributing factors}

Social connectedness or relationships influence mental health by stress-buffering and the mechanisms of the main effect [26-29]. Stress buffering assumes the association of social integration with the psychological health of individuals under stress conditions. In contrast, the main effects model is based on the belief that social ties improve mental health irrespective of the presence or absence of stress [26-29]. Stressors, including impaired physical health status, reduced mobility, lack of independence, the presence of comorbid conditions, retirement, confinement to home, fear of losing close relative, spouse bereavement, are cited as contributing factors of social isolation and adverse psychological health outcomes among the older adults [27-29]. In the context of COVID-19, the grief of losing loved ones, fear of dying with no family members around are the amplifying factors of social isolation [23, 2729]. Given the high contagious nature of the SARS-CoV-2, hospitals and nursing institutions have restricted the entry of the visitors, resulting in older adults dying in isolation, leaving behind their partners with the agony of loss and separation $[28,29]$. Due to the high prevalence of COVID-19, and the interplay between biological, environmental, and social factors, the impact on the psychological well-being of older people is likely to escalate in the foreseeable future. Therefore, addressing the social, healthcare, and emotional needs 
of the older population should take precedence to improve the psychological health outcomes.

\subsection{Shielding, social isolation, and healthcare utilization}

As an indirect benefit, shielding may help in reducing the demand for hospital beds by mitigating COVID-19 transmission among individuals with pre-existing chronic conditions [7]. More often, chronic conditions require hospital admissions for performing specialized treatments, thus increasing health care utilization and associated expenditures [30]. Besides, cognitive impairment and depressive symptoms, resulting from chronic loneliness, were reported as significant sources of increased hospital utilization trends among the older population [30]. Nearly 1 in 3 older adults found to have chronic loneliness and had multiple physician offices, visits, emergency visits, and inpatient hospital stays [25-31]. Socially isolated individuals, especially older adults, tend to utilize more medical services to stimulate interpersonal interactions for satisfying their social needs [31].

\subsection{The spectrum of psychological morbidities}

COVID-19 has the potential to trigger a myriad of mental health problems among older adults. Some of the salient ones are discussed here. The first one is depression, which is characterized by low mood, diminished interest in activities, loss or gain of weight, decrease or increase in appetite, sleep irregularities, psychomotor agitation or retardation, tiredness, feelings of worthlessness accompanied by unwarranted guilt, reduced ability to think or focus, being indecisive, recurrent thoughts of death, suicidal ideation or a suicide attempt with a particular plan [32]. Several reports revealed higher levels of depression among older adults during COVID-19 [33, 34]. The second one is the potential precipitation of manic episodes in bipolar patients [34]. These episodes include a long period of joy or excessive happiness, extreme irritability, agitation, talking rapidly, jumping from one idea to another, having racing thoughts, being easily distractible, increasing goal-directed activities, such as taking on new projects, being restless, sleeping less than usual, having an unrealistic belief in one's abilities, and impulsive behavior such as spending sprees, impulsive sex, and hasty business investments [35]. The third set of mental health problems can manifest as anxiety disorders, some of which are particularly relevant during COVID-19, including panic disorder and agoraphobia, posttraumatic stress disorder (PTSD), and generalized anxiety disorder. Bouts of anxiety or panic attacks are discrete periods of intense fear or discomfort that develops abruptly and peaks within 10 minutes. Additionally, panic disorder is accompanied by the subjective feelings of heart racing, shaking, dizziness, shortness of breath, sometimes chest pain, a fear of losing control, fear of dying, derealisation, depersonalization, dizziness, and paraesthesia [32]. PTSD is related to a traumatic experience and is characterized by three actions: re-experiencing the trauma, avoiding stimuli related to trauma, and experiencing symptoms of increased autonomic activation such as insomnia, irritability, and hypervigilance [35]. Finally, generalized anxiety disorder is associated with frequent and lasting worry, which is not in synchrony with the actual circumstances. Anxiety can get accentuated among older adults during COVID-19 [35, 36].

\subsection{Psychosocial impact of COVID-19 among older adults}

COVID-19 pandemic has a devastating effect on the psychological well-being of older adults. According to the national survey of 1468 U.S. adults, the prevalence of psychological distress among older adults ( $>55$ years) was $7.3 \%$, compared to the $3.9 \%$ reported during pre-pandemic circumstances in 2018 [36, 37]. Reportedly, loneliness has increased by 3\% during COVID-19, which suggests that loneliness is not the sole risk factor of psychological morbidities [36, 37]. Another Spain based study cited loss of loved ones, hospitalization of a family member, fear of dying, financial insecurity, low socioeconomic status, and raial disparities as potential risk factors, which may be attributed to the greater psychological distress among older adults [28, 29, 36, 37]. In the United Kingdom, the prevalence of psychological distress increased from $18.9 \%$ to $27.3 \%$, of which individuals with pre-existing chronic conditions, low-income households, and those belong to Asian ethnicity form a sizable proportion [36, 37]. It is important to emphasize that these findings were only limited to community-dwelling older adults; the psychological impact of COVID-19 among institutionalized older adults remains the subject of investigation till today.

\subsection{Risk factors}

The impact on physical health is closely tied with multiple aspects of the psychological constructs [22-24]. For instance, older adults with debilitating conditions may experience loss of autonomy and a decline in social interactions. Given the physical limitations, they lose the ability to engage in social activities, which eventually reduces the size of their social support system. In determining the risk factors of mental health in the context of COVID-19, one should be mindful of accounting potential effect modifiers, mediators, and confounders such as a change in lifestyle or daily routine, type of living arrangement (residential vs. institution), fear of losing loved ones, fear of dying, lack of economic resources, and limited communication capabilities, etc.[22, 26, 28, 29] The exploration of these factors marks avenues for future research to inform policies aimed to promote the psychological wellbeing of older adults in the wake of COVID-19.

\subsection{Influence of COVID-19 on social capital}

Social capital is a sociological construct that explains the functioning of social groups using two components of community participation that entails what people do (such as contacts with friends, neighbors, civic engagement, etc.) and social cohesion consisting of what people feel (such as belongingness, social trust, cooperation, etc.) [38-40]. This construct is especially vital for the well-being of older populations [4042]. However, the COVID-19 pandemic has lowered both the community participation and social cohesion aspects of social capital, thereby negatively affecting the well-being, especially 
among older adults. There is a need to enhance the feeling of community participation and social cohesion among the older population during the COVID-19 crisis. Social cohesion can be improved by making phone calls, using online forums and listservs, utilizations of text messaging, telepsychiatry services, and other such means.

\subsection{Prevention and treatment of mental health problems for older adults in the COVD-19 pandemic}

Primary prevention strategies of mental health problems in older adults during COVID-19 interventions should aim to build positive factors such as hardiness, sense of coherence, optimism, social support, self-esteem, and self-efficacy, physical activity [42-46]. These can be delivered through webbased educational interventions by mental health educators or psychiatrists through telepsychiatry. For secondary prevention of mental health problems in older adults during COVID19, screening and early treatments are essential, for these telepsychiatry interventions are vital, followed by psychopharmacological and psychotherapeutic interventions. Details of psychopharmacological and psychotherapeutic interventions are beyond the scope of this commentary. However, it cannot be overemphasized that telepsychiatry can play a pivotal role in addressing the gaps both in the delivery of primary and secondary prevention of mental health problems among older adults.

\subsection{Strategies to improve social connectivity}

First, it is essential to understand that "physical distancing" and "social distancing" are not interchangeable terms. It is possible to practice physical distancing while being socially integrated. Public health interventions to build communitybased and family networks for providing social and emotional support will be beneficial in improving the psychological wellbeing of the older population. The association of social support with improved mental health has already been acknowledged by previous studies [46, 47]. Also, there is enough supporting evidence, which emphasizes the effectiveness of peer support groups among the older population for satisfying the social needs of those experiencing loneliness [47, 48]. Access to telepsychiatry services with psychological assistance tools (like talk therapy) can be beneficial in addressing the psychological needs and help in improving the mental wellbeing of the older population. Access to digital technologies and increased participation of older people in the digital society will help in strengthening social inclusion among the older population. The gap in computer literacy across generations (also known as the digital divide) can pose the challenge, for which intergenerational technology learning can be viewed as a potential solution [47-49]. The evidence-based interventions using the fourth-generation Multi-Theory Model (MTM) can also be developed to increase the uptake of technology among older adults [50].

\subsection{Challenges and barriers for the adoption of telepsychiatry}

Telepsychiatry (or e-psychiatry) services have helped people to access psychological assistance tools (e.g., cognitive behavioral therapy) while maintaining a pharmaceutical medication regimen among patients utilizing psychotropic drugs to support mental well-being [51]. However, barriers in the adoption of telepsychiatry vary widely worldwide [52]. The principal barriers include technological issues, resistance to change, cost, age (which is relevant to your topic), and level of digital literacy, time, socioeconomic status, level of education, digital literacy, language barriers, unavailability of electronic devices or phones among patients, and internet connectivity and resistance to change identified by previous studies conducted in the U.S., U.K., Belgium, Australia, Netherlands, Africa, Iran, Saudi Arabia, and India [53-60]. One of the main barriers to telepsychiatry is ensuring prospective patients have access to internet-accessible technology and poor internet connectivity or lacking access completely; it a significant obstacle in telemedicine.

A 2017 survey by the Pew Research Center exploring technology use among older adults found that nearly $51 \%$ of older adults in the U.S. (aged 65 and older) reported having access to high-speed broadband [61]. Technology adoption varied significantly with socioeconomic status or income status [61, 62]. Nearly $87 \%$ of older adults who are affluent had internet access compared to only $27 \%$ among those with lower income $(<$ 30,000 /year) [61]. The level of income was positively correlated with the ownership of smartphones [61]. Socioeconomic risk factors affect older adults more than younger adults due to the fixed-income constraints faced by many older adults, who may no longer be working. Many retired or widowed older adults may not have enough money to access internet-capable technology. Resistance to technology is another factor that influences the rates of technology acceptance and utilization [63].

People living in urban areas have fewer barriers to health services than those living in rural areas. Rural-dwelling older adults will have less access to physicians, must travel much farther for health services, and may also be lacking access to community mental health services [63]. Also, those who are living in rural areas are less likely than those in urban areas to access the internet due to technological access limitations [60]. In the U.S., 19 million people living in rural or tribal areas ( $6 \%$ of the population), are lacking access to high-speed broadband internet, according to the U.S. Federal Communications Commission (FCC) report of 2020 [64]. Consistent with this report, per the Telecom Regularity Authority of India, only $28 \%$ of people residing in rural areas had access to the internet, illustrating the challenge posed in telemedicine [60]. These financial and technological access issues are substantial barriers that require infrastructure investments and technology acquisition assistance that is not currently available in the middle of the COVID-19 pandemic.

Other issues include stigmatization, privacy, and digital literacy. Particularly with the telepsychiatry, accessing mental health care still has a stigma for many people. Some older adults may feel uncomfortable using telepsychiatry for their 
mental health needs due to concerns over privacy, confidentiality, or quality of the interaction [60]. But some patients may feel more comfortable sharing personal information from the privacy of their own homes, instead of in a doctor's office setting that may feel less private. Also, digital literacy and computer use skills may not be as advanced in this population, potentially requiring assistance in setting up the hardware needed for effective telemedicine appointments (such as a computer, webcam, and microphone) [61]. It is essential to understand these barriers and challenges to improve support for older adults around the use of technology and telemedicine. Intergenerational pieces of training and peer support interventions will play instrumental roles in overcoming these obstacles.

\section{Conclusions}

The problem of social isolation and loneliness are the longstanding public health concerns of the older population. Social distancing may limit the transmission of COVID-19, but its impact on psychological health is harmful. Improving meaningful social connectedness through peer support groups and efficient public health monitoring of mental illness are the potential strategies to increase social inclusion among the older population. Adoption and uptake of telepsychiatry health care and remote counseling support services need to be exercised to improve the mental well-being of older adults living in the shadow of the pandemic with fear, anxiety, depression, and social disconnectedness. Addressing the long-standing unmet needs of social integration or connectedness and improving social capital among older adults becomes an urgent priority during the COVID-19 crisis and for the recovery period ahead. Fourth-generation theory models should be utilized to develop an evidence-based intervention to increase digital literacy among the older population.

\section{ACKNOWLEDGMENTS}

The authors would like to thank all the peer reviewers and editors for their opinions and suggestions. In addition, we wish to express our gratitude to Mr. Ravi Batra for his assistance in formatting and proofreading.

\section{CONFLICT OF INTEREST}

The authors declare that there is no conflict of interest regarding the publication of this article.

\section{AUTHORS CONTRIBUTIONS}

K.B. conceived the idea. K.B., A.M., M.S., performed independent literature searches and wrote the manuscript. All authors contributed to editorial changes in the manuscript. All authors read and approved the final manuscript.

\section{FINANCIAL DISCLOSURE}

This research did not receive any specific grants from funding agencies in the public, commercial, or not-for-profit sectors.

\section{REFERENCES}

[1] WHO Coronavirus Disease (COVID-19) Dashboard. World Health Organization website (2020a). https : / / covid19. who. int/.

[2] COVID-19 strategy update - 14 April 2020. World Health Organization website (2020b). https://www.who.int/publications/i/item/ covid-19-strategy-update---14-april-2020.

[3] Coronavirus disease 2019 (COVID-19) associated hospitalizations surveillance network (COVID-NET). Centers for Disease Control and Prevention website (2020a). Retrieved from https://www.cdc.gov/coronavirus/2019-ncov/need-extraprecautions/older-adults.html.

[4] Severe Outcomes Among Patients with Coronavirus Disease 2019 (COVID-19) - United States, February 12-March 16. Centers for Disease Control and Prevention website (2020b). https://www.cdc.gov/ mmwr/volumes/69/wr/mm6912e2.htm.

[5] Shahid Z, Kalayanamitra R, McClafferty B, et al. COVID-19 and older adults: what we know. J Am Geriatr. Soc. 2020; 68: 926-929.

[6] Report of the WHO-China Joint Mission on Coronavirus Disease 2019 (COVID-19). WHO-China Joint Mission website (2020). https: //www. who.int/docs/default-source/coronaviruse/whochina-joint-mission-on-covid-19-final-report.pdf.

[7] Clark A, Jit M, Warren GC. Global, regional, and national estimates of the population at increased risk of severe COVID-19 due to underlying health conditions in 2020: a modelling study. Lancet Glob. Health. 2020;8:e1003-e1017.

[8] Sadighi Akha A.A. Aging and the immune system: An overview. J. Immunol. Methods. 2018;463:21-26.

[9] Chow N, Fleming DK, Gierke R. Preliminary estimates of the prevalence of selected underlying health conditions among patients with coronavirus disease 2019 - United States, February 12-March 28, 2020. MMWR Morb Mortal Wkly Rep. 2020; 69:382-386.

[10] United Nations. (2020). Policy brief: The impact of COVID-19 on older persons. Retrieved from United Nations website: https: //unsdg. un .org/sites/default/files/2020-05/PolicyBrief-The-Impact-of-COVID-19-on-0lder-Persons . pdf.

[11] D'Adamo H, Yoshikawa T, Ouslander JG. Coronavirus disease 2019 in geriatrics and long-term care: The ABCDs of COVID-19. J Am Geriatr Soc. 2020;68:912-917.

[12] Feaster M, Goh Y. High proportion of asymptomatic SARS-Cov-2 infections in 9 long-term care facilities, Pasadena, California, USA, April 2020. Emerg Infect Dis. 2020;26. doi:10.3201/eid2610.202694.

[13] Daoust JF. Elderly people and responses to COVID-19 in 27 Countries. PLoS One. 2020;15:e235590.

[14] Armitage R, Nellums LB. COVID-19 and the consequences of isolating the elderly. Lancet Public Health. 2020;5:e256.

[15] Van Orden KA, Bower E, Lutz J, et al. Strategies to Promote Social Connections Among Older Adults During 'Social Distancing' Restrictions. Am J Geriatr Psychiatry. 2020. doi: 10.1016/j.jagp.2020.05.004.

[16] Melchiorre MG, Chiatti C, Lamura G, et al. Social support, socioeconomic status, health and abuse among older people in seven European countries. PLoS One. 2013;8:e54856.

[17] Jaremka LM, Andridge RR, Fagundes CP, et al. Pain, depression, and fatigue: loneliness as a longitudinal risk factor. Health Psychol. 2014;33:948-957.

[18] Steptoe A, Shankar A, Demakakos P, Wardle J. Social isolation, loneliness, and all-cause mortality in older men and women. Proc Natl Acad Sci U S A. 2013;110:5797-5801.

[19] National Institute of Ageing (2019). Social isolation and loneliness in older people pose health risks. Retrieved from https: //www.nia.nih.gov/news/social-isolation-lonelinessolder-people-pose-health-risks.

[20] Santini ZI, Jose PE, York Cornwell E, et al. Social disconnectedness, perceived isolation, and symptoms of depression and anxiety among older Americans (NSHAP): a longitudinal mediation analysis. Lancet Public Health. 2020;5:e62-e70.

[21] Gustavsson J, Beckman L. Compliance to Recommendations and Mental 
Health Consequences among elderly in Sweden during the initial phase of the COVID-19 pandemic-a cross sectional online survey. Int J Environ Res Public Health. 2020;17:5380.

[22] Tough H, Siegrist J, Fekete C. Social relationships, mental health and well-being in physical disability: a systematic review. BMC Public Health. 2017;17:414.

[23] López J, Perez-Rojo G, Noriega C, et al. Psychological well-being among older adults during the COVID-19 outbreak: a comparative study of the young-old and the old-old adults. Int Psychogeriatr. 2020;1-6. doi:10.1017/S1041610220000964.

[24] Ryff CD. Psychological well-being revisited: advances in the science and practice of eudaimonia. Psychother Psychosom. 2014;83:10-28.

[25] Gerst EK, Jayawardhana J. Loneliness as a public health issue: The impact of loneliness on health care utilization among older adults. Am. J. Public Health. 2015;105:1013-1019.

[26] Cacioppo JT, Cacioppo S. The growing problem of loneliness. Lancet (London, England). 2018;391:426.

[27] Fässberg MM, Cheung G, Canetto SS, et al. A systematic review of physical illness, functional disability, and suicidal behaviour among older adults. Aging Ment Health. 2015;20:166-194.

[28] Wegleitner K, Schuchter P, Prieth S. 'Ingredients' of a supportive web of caring relationships at the end of life: findings from a community research project in Austria. Sociol Health Illn. 2020;42:987-1000.

[29] Abdi S, Spann A, Borilovic J, et al. Understanding the care and support needs of older people: A scoping review and categorisation using the WHO international classification of functioning, disability and health framework (ICF). BMC Geriatr. 2019;19:1-15.

[30] Carr D, Boerner K, Moorman S. Bereavement in the time of coronavirus: Unprecedented challenges demand novel interventions. J Aging Soc Policy. 2020;32:425-431.

[31] Molloy GJ, McGee HM, O'Neill D, et al. Loneliness and emergency and planned hospitalizations in a community sample of older adults. J. Am Geriatr Soc. 2010;58:1538-1541.

[32] Perissinotto CM, Stijacic CI, Covinsky KE. Loneliness in older persons. Arch Intern Med. 2012.

[33] American Psychiatric Association (APA). Diagnostic and statistical manual of mental disorders (DSM-5). 5th ed. American Psychiatric Publishing; 2013.

[34] Choi EPH, Hui BPH, Wan EYF. Depression and Anxiety in Hong Kong during COVID-19. Int J Environ Res Public Health. 2020;17:3740.

[35] Van Rheenen TE, Meyer D, Neill E, et al. Mental health status of individuals with a mood-disorder during the COVID-19 pandemic in Australia: Initial results from the COLLATE project. J Affect Disord. 2020;275:69-77.

[36] Sharma M, Branscum P. Foundations of mental health promotion. 2nd ed. Jones and Bartlett; 2020.

[37] Huang Y, Zhao N. Generalized anxiety disorder, depressive symptoms and sleep quality during COVID-19 outbreak in China: a web-based cross-sectional survey. Psychiatry Res. 2020;288:112954.

[38] McGinty EE, Presskreischer R, Han H, et al. Psychological Distress and Loneliness Reported by U.S. Adults in 2018 and April 2020. JAMA. 2020;324:93-94.

[39] Pierce M, Hope H, Ford T, et al. Mental health before and during the COVID-19 pandemic: a longitudinal probability sample survey of the U.K. population. Lancet Psychiatry. 2020;S2215-0366:30308-30304.

[40] Isbel ST, Berry HL. Social capital, health, and elderly driver status. Yale J Biol Med. 2016;89: 87-90.

[41] Putnam RD. Bowling alone: the collapse and revival of American community. Simon and Schuster. 2020.

[42] Alvarez EC, Kawachi I, Romani JR. Family social capital and health - a systematic review and redirection. Sociol Healt Illn. 2017; 39:5-29.

[43] Lee S, Jung M. Social capital, community capacity, and health. The Health Care Manag. 2018;37:290-298.

[44] Goodman-Casanova JM, Dura-Perez E, Guzman-Parra J, et al. Telehealth home support during COVID-19 confinement for community-dwelling older adults with mild cognitive impairment or mild dementia: survey study. J Med Internet Res. 2020;22:e19434.
[45] Kanekar A, Sharma M. COVID-19 and mental well-being: Guidance on the application of behavioral and positive well-being strategies. Healthcare. 2020;8:1-7.

[46] Hernández-Padilla JM, Granero-Molina J, Ruiz-Fernández MD, et al. Design and Psychometric Analysis of the COVID-19 Prevention, recognition and home-management self-efficacy scale. Int J Environ Res Public Health. 2020;17:4653.

[47] Jiménez-Pavón D, Carbonell-Baeza A, Lavie C.J. Physical exercise as therapy to fight against the mental and physical consequences of COVID19 quarantine: special focus in older people. Prog Cardiovasc Dis. 2020;63:386-388.

[48] Gong X, Ni Z, Wu B. The mediating roles of functional limitations and social support on the relationship between vision impairment and depressive symptoms in older adults. Ageing Soc. 2018; 40:465-479.

[49] Hertha BS, Krašovec SJ, Formosa M. Learning across generations in Europe: Contemporary issues in older adult education. Springer. 2014.

[50] Sharma M. Multi-theory model (MTM) for health behaviour change. WebMed Central Behaviour. 2015;6:WMC004982.

[51] Zhou X, Snoswell CL, Harding LE, et al. The Role of Telehealth in Reducing the Mental Health Burden from COVID. Telemed J E Health. 2020;26:377-379.

[52] Kruse CS, Karem P, Shifflett K, et al. Evaluating barriers to adopting telemedicine worldwide: A systematic review. J Telemed Telecare. 2018; 24:4-12.

[53] Plaete J, Crombez G, DeSmet A, et al. What do general practitioners think about an online self-regulation programme for health promotion? Focus group interviews. BMC Fam Pract. 2015;16:1-11.

[54] Schwarz F, Ward J, Willcock S. E-Health readiness in outback communities: an exploratory study. Rural Remote Health. 2014;14:2871.

[55] Van Deursen A.J., van Dijk JA. Internet skills performance tests: are people ready for eHealth? J Med Internet Res. 2011;13: e35-e35.

[56] Bigna JJ, Noubiap JJ, Plottel CS, et al. Barriers to the implementation of mobile phone reminders in pediatric HIV care: a pre-trial analysis of the Cameroonian MORE CARE study. BMC Health Serv Res. 2014; 14:1-7.

[57] Mohammadzadeh N, Safdari R, Rahimi A. Cancer care management through a mobile phone health approach: key considerations. Asian Pac J Cancer Prev. 2013;14:4961-4964.

[58] El-Mahalli AA, El-Khafif SH, Al-Qahtani MF. Successes and challenges in the implementation and application of telemedicine in the eastern province of Saudi Arabia. Perspect Health Inf Manag. 2012;9:1-27.

[59] Scholl J, Syed-Abdul S, Ahmed LA. A case study of an EMR system at a large hospital in India: challenges and strategies for successful adoption. J Biomed Inform. 2011;44:958-967.

[60] Malathesh BC, Gowda GS, Kumar CN, et al. Response to: Rethinking online mental health services in China during the COVID-19 epidemic. Asian J. Psychiatry. 2020;51;102105.

[61] Anderson M, Perrin A. Pew Research Center Internet and Technology. May 17, 2017. https://www.pewresearch.org/internet/2017/ 05/17/technology-use-among-seniors/.

[62] Yao H, Chen JH, Xu YF. Rethinking online mental health services in China during the COVID-19 epidemic. Asian J Psychiatr. 2020;50:102015.

[63] Peters DJ. Community susceptibility and resiliency to COVID-19 across the rural-urban continuum in the United States. J Rural Health. 2020;36:446-456.

[64] Eighth Broadband Progress Report. Federal Communications Commission website. https://www.fcc.gov/reportsresearch/reports/broadband-progress-reports/eighthbroadband-progress-report.

How to cite this article: Kavita Batra, Amanda Elizabeth Morgan, Manoj Sharma. COVID-19 and Social Isolation Endangering Psychological Health of Older Adults: Implications for Telepsychiatry. Signa Vitae. 2020;16(2):14-19. doi:10.22514/sv.2020.16.0070. 